\title{
On Error Probability for Non-coherent MIMO Channels in the Wideband Regime
}

\author{
Siddharth Ray, Muriel Médard and Lizhong Zheng \\ Laboratory for Information and Decision Systems \\ Massachusetts Institute of Technology \\ Cambridge, MA 02139, USA. \\ Email: sray@mit.edu, medard@mit.edu, lizhong@mit.edu
}

\begin{abstract}
We consider a multiple-input, multiple-output (MIMO) wideband Rayleigh block fading channel where the channel state is unknown to both the transmitter and the receiver and there is only an average power constraint on the input. We compute the error probability and study its dependence on receive signal-to-noise ratio (SNR), number of transmit and receive antennas and coherence length. We show that error probability decays inversely with coherence length and exponentially with the product of the number of transmit and receive antennas. Moreover, channel outage dominates error probability in the wideband regime. We also show that the critical as well as cut-off rates are much smaller than channel capacity in this regime.
\end{abstract}

\section{INTRODUCTION}

Multiple-input, multiple-output (MIMO) systems have been known to improve considerably performance of wireless systems in terms of reliability as well as throughput, without requiring additional resources such as bandwidth and power. Motivated by the ever increasing demand for higher wideband wireless data rates, we consider multiple antenna communication over a wideband wireless channel. While communicating over a wideband channel, the available power is spread over a large number of degrees of freedom and, the received signalto-noise ratio (SNR) per degree of freedom is low. Hence, while studying these channels, we need to focus on the low SNR regime. We will therefore use the terms "wideband" and "low SNR" interchangeably, with the understanding that the latter refers to the SNR per degree of freedom.

MIMO channels with perfect channel state information (CSI) at the receiver (coherent channel) but no CSI at the transmitter were first studied from a capacity point of view in [2], [5]. When CSI is unavailable at the receiver also (non-coherent channel), the structure of the optimal input matrix is obtained in [4]. The coherent and non-coherent capacities are computed in [2] and [8], respectively, when the received SNR is high.

For the Rayleigh block fading non-coherent MIMO channel, [8] shows that:

$$
\lim _{\mathrm{SNR} \rightarrow 0} \frac{C(\mathrm{SNR})}{\mathrm{SNR}}=r,
$$

where, $r$ is the number of receive antennas, SNR is the average signal-to-noise ratio per degree of freedom at each receive antenna and $C(\mathrm{SNR})$ is the non-coherent capacity per degree of freedom. The capacity can thus be expressed as:

$$
C(\mathrm{SNR})=r \mathrm{SNR}+o(\mathrm{SNR}) \text { nats/channel use }
$$

and is thus a linear function only in the limit of low SNR. As SNR increases from 0, capacity increases in a sublinear fashion, showing that low SNR communication is power efficient. We use the MIMO sublinear capacity term definition introduced in [14]:

$$
\Delta^{(t, r)}(\mathrm{SNR}) \triangleq r \mathrm{SNR}-C(\mathrm{SNR}) \text { nats/channel use, }
$$

where, $t$ and $r$ are the number of transmit and receive antennas, respectively. This term is computed in [14] as

$$
\Delta^{(t, r)}(\mathrm{SNR})=\frac{r(r+t)}{2 t} \mathrm{SNR}^{1+\alpha}+o\left(\mathrm{SNR}^{1+\alpha}\right),
$$

where, $\alpha \in(0,1]$ is related to the coherence length, SNR and antennas as $l \sim \frac{t^{2}}{(r+t)^{2}}$ SNR $^{-2 \alpha}$.

In this paper, we analyze the error probability for the noncoherent wideband (low SNR) MIMO channel. The behavior of error probability for the coherent [3], [11] as well as noncoherent [6], [9] MIMO channels has been well studied in the high SNR regime. For the coherent MIMO channel with coherence length 1 symbol, the error exponent is computed by Telatar [5] for any SNR. The behavior of the error exponent for the non-coherent MIMO channel in the low SNR regime has recently been considered by $\mathrm{Wu}$ and Srikant in [12]. Their analysis considers the linear capacity term, $r \mathrm{SNR}$, and the error exponent is computed by fixing the coherence length and letting SNR tend to 0 .

Our consideration of the effect of the interaction among SNR, number of transmit and receive antennas and coherence length, on the error probability, yields a more detailed characterization of the error probability behavior than described in [12]. Our analysis shows that in the low SNR regime, the critical rate as well as the cut-off rate are much smaller than the channel capacity. Moreover, the error probability decays inversely with coherence length. We introduce the notion of "diversity" in the low SNR regime and use it to show that error probability decays exponentially with the product of the number of transmit and receive antennas. Hence, in terms of reliability in the wideband regime, transmit antennas have the same importance as receive antennas. In the high SNR regime, it is well known that outage dominates the error probability. Our 
analysis shows that this is true even at low SNR, i.e., channel outage dominates the error probability at low SNR.

Let us establish notation that will be used in the rest of the paper. The bold type will be used to denote random quantities whereas normal type will be used to denote deterministic ones. Matrices will be denoted by capital letters and the scalar or vector components of matrices will be denoted using appropriate subscripts. Vectors will be represented by small letters with an arrow over them. All vectors are column vectors unless they have a ${ }^{T}$ superscript. Scalars will be represented by small letters only. The superscript ${ }^{\dagger}$ will be used to denote the complex conjugate transpose.

The rest of the paper is organized as follows: We describe the channel model in section II and present the error probability results in section III. We conclude in section IV.

\section{MOdeL}

We model the wideband channel as a set of $N$ parallel narrowband channels. In general, the narrowband channels will be correlated. We restrict our analysis in this paper to channels having independent and identical statistics. We also assume that the coherence bandwidth is much larger than the bandwidth of the narrowband channel. Hence, each narrowband channel is modeled as being flat faded. From [13], we see that low SNR channels are robust to reasonable modeling assumptions. Hence, the results for a more precise MIMO channel model will not differ significantly from that of the simple model we consider in this paper.

Using the sampling theorem, the $m^{\text {th }}$ narrowband channel at symbol time $k$ can be represented as:

$$
\overrightarrow{\mathbf{y}}[k, m]=\mathbf{H}[k, m] \overrightarrow{\mathbf{x}}[k, m]+\overrightarrow{\mathbf{w}}[k, m],
$$

where $\mathbf{H}[k, m], \overrightarrow{\mathbf{x}}[k, m], \overrightarrow{\mathbf{w}}[k, m]$ and $\overrightarrow{\mathbf{y}}[k, m]$ are the channel matrix, input vector, noise vector and output vector, respectively, for the $m^{\text {th }}$ narrowband channel at symbol time $k$. The pair $(k, m)$ may be considered as an index for the timefrequency slot, or degree of freedom, to communicate. We denote the number of transmit and receive antennas by $t$ and $r$, respectively. Hence, $\overrightarrow{\mathbf{x}}[k, m] \in \mathcal{C}^{t}$ and $\overrightarrow{\mathbf{y}}[k, m], \overrightarrow{\mathbf{w}}[k, m] \in \mathcal{C}^{r}$. The channel matrix $\mathbf{H}[k, m]$ is a $r \times t$ complex matrix. The entries of the channel matrix are i.i.d zero-mean complex Gaussian, with independent real and imaginary components. Equivalently, each entry of $\mathbf{H}[k, m]$ has uniformly distributed phase and Rayleigh distributed magnitude. We thus model a Rayleigh fading channel with enough separation within the transmitting and receiving antennas to achieve independence in the entries of $\mathbf{H}[k, m]$. The channel matrix is unknown at the transmitter and the receiver. However, its statistics are known to both. The noise vector $\overrightarrow{\mathbf{w}}[k, m]$ is a zero-mean Gaussian vector with the identity as its covariance matrix. Thus, $\overrightarrow{\mathbf{w}}[k, m] \sim \mathcal{C N}\left(\overrightarrow{0}, I_{r}\right)$. Since the narrowband channels are assumed to be independent, we will omit the narrowband channel index, $m$, to simplify notation. The capacity of the wideband channel with power constraint $P$ is thus $N$ times the capacity of each narrowband channel with power constraint $P / N$. Hence, we can focus on the narrowband channel alone.
We further assume a block fading channel model, i.e., the channel matrix is random but fixed for the duration of the coherence time of the channel, and is i.i.d across blocks. Hence, we may omit the time index, $k$, and express the narrowband channel within a coherence block of length $l$ symbols as:

$$
\mathbf{Y}=\mathbf{H X}+\mathbf{W},
$$

where, $\mathbf{X} \in \mathcal{C}^{t \times l}$ has entries $\mathbf{x}_{i j}, i=1, \ldots, t, j=1, \ldots, l$, being the signals transmitted from the transmit antenna $i$ at time $j ; \mathbf{Y} \in \mathcal{C}^{r \times l}$ has entries $\mathbf{y}_{i j}, i=1, \ldots, r, j=1, \ldots, l$, being the signals received at the receive antenna $i$ at time $j$; the additive noise $\mathbf{W}$ has i.i.d. entries $\mathbf{w}_{i j}$, which are distributed as $\mathcal{C N}(0,1)$. The input $\mathbf{X}$ satisfies the average power constraint

$$
\frac{1}{l} E\left[\operatorname{trace}\left\{\mathbf{X X}^{\dagger}\right\}\right]=\mathrm{SNR} \text {. }
$$

As $N$ tends to $\infty$, SNR tends to 0 , and the narrowband channel is in the low SNR regime.

\section{ERROR PROBABILITY DERIVATION}

From [14], we see that a non-coherent MIMO channel with coherence length

$$
\frac{t^{2}}{(r+t)^{2}} \mathrm{SNR}^{-2 \nu}<l \leq \frac{t^{2}}{(r+t)^{2}} \mathrm{SNR}^{-2(\nu+\epsilon)},
$$

where, $\nu>0$ and $\epsilon \in(0, \min \{1, \nu\})$, has capacity

$$
\begin{aligned}
C(\mathrm{SNR})=\quad r \mathrm{SNR}-\frac{r(r+t)}{2 t} & \mathrm{SNR}^{1+\min \{1, \nu\}} \\
& +O\left(\mathrm{SNR}^{1+\min \{1, \nu\}+\epsilon}\right) .
\end{aligned}
$$

The channel capacity and coherence length are related through $\nu$. The expression, $\min \{1, \nu\}$, is the coherence level and is an indicator of the amount of coherence in the channel. A detailed treatment of coherence level can be found in [13], [14].

Reference [14] introduces a near-capacity achieving signaling scheme - the Peaky Gaussian signaling scheme. We present this scheme here for completeness.

For a channel with coherence length

$$
l=\frac{t^{2}}{(r+t)^{2}} \mathrm{SNR}^{-2 \nu}, \quad \nu>0,
$$

transmit in only $\delta(\mathrm{SNR})=\mathrm{SNR}^{1-\min \{1, \nu\}}$ fraction of the blocks. Since the power is concentrated in only over a fraction of the blocks, the signal to noise ratio for the blocks used for transmission increases to $\mathrm{SNR}_{b}$, where

$$
\mathrm{SNR}_{b} \triangleq \frac{\mathrm{SNR}}{\delta(\mathrm{SNR})}=\mathrm{SNR}^{\min \{1, \nu\}}
$$

In the blocks chosen for transmission, let the entries of the input matrix $\mathbf{X}$ be i.i.d $\mathcal{C N}\left(0, \frac{\mathrm{SNR}_{b}}{t}\right)$. Note that, as we increase the coherence length, the fraction of blocks that we transmit increases from SNR to 1. Therefore, the signaling changes from a peaky to a continuous one.

In this paper, we compute the average block error probability 
for the non-coherent MIMO channel, $P_{\text {error }}^{\text {block }}$, when maximumlikelihood decoding is used at the receiver with Peaky Gaussian input signaling. This error probability can be expressed as

$$
P_{\text {error }}^{\text {block }}=\operatorname{Pr}(\text { error } \mid \mathcal{B}) \operatorname{Pr}(\mathcal{B})+\operatorname{Pr}(\text { error } \mid \overline{\mathcal{B}}) \operatorname{Pr}(\overline{\mathcal{B}})
$$

where, $\mathcal{B}$ is the event that the coherence block is used for transmission. Since we use Peaky Gaussian signaling and the receiver is assumed to have perfect knowledge of the blocks that are being used for transmission, we have

$$
\begin{aligned}
\operatorname{Pr}(\mathcal{B}) & =\delta(\text { SNR }), \\
\operatorname{Pr}(\text { error } \mid \overline{\mathcal{B}}) & =0 .
\end{aligned}
$$

Hence,

$$
P_{\text {error }}^{\text {block }}=\delta(\mathrm{SNR}) \cdot \operatorname{Pr}(\text { error } \mid \mathcal{B})
$$

If we consider the input matrix transmitted in a block, $\mathbf{X}$, as a super symbol of dimension $t \times l$, the channel is memoryless, since, for each use of the channel an independent realization of $\mathbf{H}$ is drawn. Hence, using the results in [1], the error probability can be bounded as

$$
\operatorname{Pr}(\operatorname{error} \mid \mathcal{B}) \leq \exp \left[-E_{r}(R)\right]
$$

where, $E_{r}(R)$ is the random coding error exponent for the super symbol channel:

$$
E_{r}(R)=\max _{\rho \in[0,1]}\left\{E_{0}(\rho)-\rho R\right\},
$$

where,

$$
E_{0}(\rho)=-\log \int\left[\int q(X) p(Y \mid X)^{\frac{1}{1+\rho}} d X\right]^{1+\rho} d Y
$$

$q(X)$ is the distribution of $\mathbf{X}, R$ is the transmission rate in nats per block used for transmission and $\mathbf{Y}$ is the channel's output matrix.

Since the signaling is Gaussian in the block used for transmission,

$$
q(X)=\frac{1}{\pi^{l t}} \exp \left[-\operatorname{trace}\left(X^{\dagger} X\right)\right] .
$$

The range of $R$ for which $E_{r}(R)$ is positive is:

$$
0 \leq R \leq \frac{l}{\delta(\mathrm{SNR})} \cdot C(\mathrm{SNR}) \triangleq C^{\text {block }}(\mathrm{SNR}),
$$

where, $C^{\text {block}}(\mathrm{SNR})$ is the non-coherent capacity per block:

$$
\begin{aligned}
C^{\text {block }}(\mathrm{SNR}) & \\
= & \frac{t^{2}}{(r+t)^{2}} \mathrm{SNR}^{-2 \nu} \\
& \cdot\left[r \mathrm{SNR}^{\min \{1, \nu\}}-\frac{r(r+t)}{2 t} \mathrm{SNR}^{2 \min \{1, \nu\}]}+\right. \\
& \quad+o\left(\mathrm{SNR}^{-2[\nu-\min \{1, \nu\}]) .}\right.
\end{aligned}
$$

The main result of this paper is summarized in the following theorem. The proof, which we omit here for brevity, is in section 4.2 of [15].

Theorem 1: The average block error probability for a noncoherent Rayleigh block fading MIMO channel, $P_{\text {error }}^{\text {block }}$, when maximum-likelihood decoding is used at the receiver can be upper bounded as:

$$
P_{\text {error }}^{\text {block }} \leq\left[\mathrm{SNR}^{1-\min \{1, \nu\}}\right] \cdot \exp \left[-E_{r}(R)\right],
$$

where,

$$
\begin{array}{rl}
E_{r}(R) & =r t \log \left(1+\frac{t \mathrm{SNR}^{-[2 \nu-\min \{1, \nu\}]}}{2(t+r)^{2}}\right)-R-o(1) \\
R \in\left[0, R_{\text {critical }}\right], & \\
= & r t \log \left(1+\frac{\rho^{*} t \mathrm{SNR}^{-[2 \nu-\min \{1, \nu\}]}}{(t+r)^{2}\left(1+\rho^{*}\right)}\right)-\rho^{*} R-o(1) \\
R \in\left[R_{\text {critical }}, C_{\mathrm{T}, \mathrm{lb}}^{\text {block }}(\mathrm{SNR})\right], \\
=o(1) & R \in\left[C_{\mathrm{T}, \text { lb }}^{\text {block }}(\mathrm{SNR}), C^{\text {block }}(\mathrm{SNR})\right], \\
=0 & R \in\left[C^{\text {block }}(\mathrm{SNR}), \infty\right),
\end{array}
$$

and

$$
\begin{aligned}
& \rho^{*}=\frac{1}{2}\left[\sqrt{1+4\left(\frac{r t}{R}-\frac{(t+r)^{2} \mathrm{SNR}^{2 \nu-\min \{1, \nu\}}}{t}\right)}-1\right] \\
& R_{\text {critical }}= r t / 2+o(1) \\
& C_{\mathrm{T}, \mathrm{lb}}^{\text {block }}(\mathrm{SNR}) \\
&=\frac{t^{2}}{(r+t)^{2}} \mathrm{SNR}^{-2 \nu}\left[r \mathrm{SNR}^{\min \{1, \nu\}}\right. \\
&-2 \frac{r(r+t)}{\sqrt{t}} \mathrm{SNR}^{\nu+\frac{\min \{1, \nu\}}{2}}-\frac{r(r+t)}{2 t} \mathrm{SNR}^{2 \min \{1, \nu\}} \\
& \quad+o\left(\mathrm{SNR}^{\left.\left.\min \left\{\nu+\frac{\min \{1, \nu\}}{2}, 2 \min \{1, \nu\}\right\}\right)\right] .}\right.
\end{aligned}
$$

\section{A. Discussion of Theorem 1}

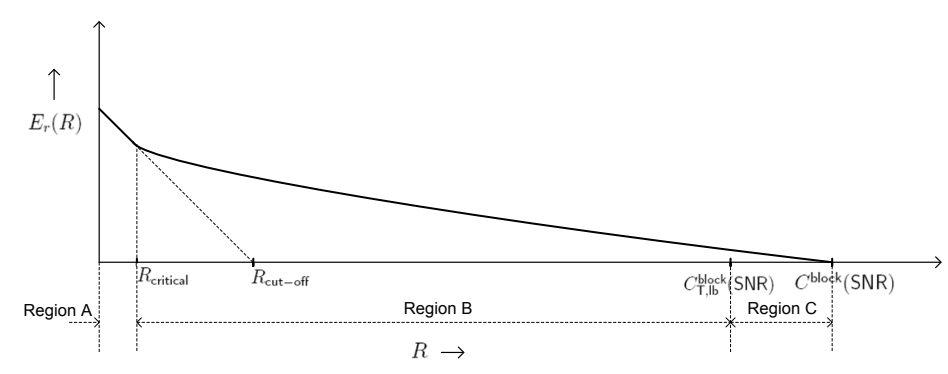

Fig. 1. Random coding error exponent for the non-coherent MIMO channel at low SNR.

Theorem 1 divides the range of rates for which $E_{r}(R)$ is positive into three regions - A, B and $\mathrm{C}$, which is illustrated 
in Figure 1. Let us consider region A: $R \in\left[0, R_{\text {critical }}\right]$. Since, $R_{\text {critical }}=O(1)$ and $C^{\text {block }}(\mathrm{SNR})=O\left(\mathrm{SNR}^{-[2 \nu-\min \{1, \nu\}]}\right)$, the critical rate is much smaller than the channel capacity:

$$
R_{\text {critical }} \ll C^{\text {block }}(\mathrm{SNR}) \text {. }
$$

Region A is an $O\left(\mathrm{SNR}^{[2 \nu-\min \{1, \nu\}]}\right)$ fraction of the capacity and is very small in the wideband regime. The cut-off rate, $R_{\text {cut-off }}$, is given by ${ }^{1}$

$R_{\text {cut }- \text { off }}=E_{r}(0) \doteq r t \cdot[2 \nu-\min \{1, \nu\}] \cdot \log \left(\frac{1}{\mathrm{SNR}}\right)$.

Since the cut-off rate is an $O\left(\operatorname{SNR}^{[2 \nu-\min \{1, \nu\}]} \cdot \log \left(\frac{1}{\operatorname{SNR}}\right)\right)$ fraction of the capacity, it is much smaller than the capacity in the wideband regime:

$$
R_{\text {cut-off }} \ll C^{\text {block }}(\mathrm{SNR}) .
$$

Hence, the critical and cut-off rates are much smaller than the capacity in the low SNR regime.

Let us consider the third region over which $E_{r}(R)$ is positive, region $\mathrm{C}: R \in\left[C_{\mathrm{T}, \mathrm{lb}}^{\text {block}}(\mathrm{SNR}), C^{\text {block}}(\mathrm{SNR})\right]$. This interval is a $\left[C^{\text {block}}(\mathrm{SNR})-C_{\mathrm{T}, \mathrm{lb}}^{\text {block }}(\mathrm{SNR})\right] / C^{\text {block }}(\mathrm{SNR})$ fraction of the capacity where,

$$
\frac{C^{\text {block}}(\mathrm{SNR})-C_{\mathrm{T}, \mathrm{lb}}^{\text {block }}(\mathrm{SNR})}{C^{\text {block }}(\mathrm{SNR})}=\left\{\begin{array}{cc}
O\left(\mathrm{SNR}^{\nu-\frac{\min \{1, \nu\}}{2}}\right) & \nu \leq \frac{3}{2} \\
o(\mathrm{SNR}) & \nu>\frac{3}{2}
\end{array}\right.
$$

Hence, region $\mathrm{C}$ is also a very small fraction of the capacity in the wideband regime. Therefore, we can conclude that it is region $\mathrm{B}: R \in\left[R_{\text {critical }}, C_{\mathrm{T}, \mathrm{lb}}^{\text {block }}(\mathrm{SNR})\right]$, that dominates the range of rates in the wideband regime.

From Theorem 1, the error probability in Region B can be expressed as:

$$
P_{\text {error }}^{\text {block }} \sim \mathrm{SNR}^{1-\min \{1, \nu\}} \cdot\left[\frac{R}{l \cdot \mathrm{SNR}^{\min \{1, \nu\}}}\right]^{r t} .
$$

To observe this, let us consider the error exponent for

$$
R=l \cdot r \mathrm{SNR}^{\kappa}, \quad \min \{1, \nu\}<\kappa<2 \nu .
$$

This rate lies in Region B and the optimum $\rho$ is

$$
\rho^{*}=O\left(\frac{1}{R}\right) .
$$

Substituting in Theorem 1, we observe

$$
E_{r}(R) \doteq r t \log \left[\frac{l \cdot \mathrm{SNR}^{\min \{1, \nu\}}}{R}\right]
$$

\footnotetext{
${ }^{1}$ Definition of $(\dot{\doteq})$ : Let $f(\mathrm{SNR})$ and $g(\mathrm{SNR})$ be functions of SNR. We denote $f(\mathrm{SNR}) \doteq g(\mathrm{SNR})$ if

$$
\lim _{\mathrm{SNR} \rightarrow 0} \frac{\log f(\mathrm{SNR})}{\log g(\mathrm{SNR})}=1 .
$$
}

For $\nu \leq 1, \operatorname{SNR}^{\min \{1, \nu\}} \propto 1 / \sqrt{l}$. Hence, for a fixed rate $R$, the error probability decays inversely with the coherence length in the following way:

$$
P_{\text {error }}^{\text {block }} \propto\left\{\begin{array}{cl}
\left(\frac{1}{l}\right)^{\frac{r t-1}{2}} & l \leq \mathrm{SNR}^{-2} \\
\left(\frac{1}{l}\right)^{r t} & l>\mathrm{SNR}^{-2}
\end{array} .\right.
$$

Let us now examine the effect of antennas on the error probability. To analyze this, we propose a definition of "diversity" in the low SNR / wideband regime.

Let $\mathcal{P}$ and $W$ be the total received power and system bandwidth, respectively. High SNR diversity, $d_{H}(W)$, is commonly defined as:

$$
d_{H}(W) \triangleq-\lim _{\mathcal{P} \rightarrow \infty} \frac{\log \left(P_{\text {error }}^{\text {bloc }}(\mathcal{P}, W)\right)}{\log (\mathcal{P})} .
$$

This definition describes the asymptotic behavior of error probability with received power, for fixed bandwidth.

In the low SNR/wideband regime, we define diversity, $d_{L}(\mathcal{P})$, as:

$$
d_{L}(\mathcal{P}) \triangleq-\lim _{W \rightarrow \infty} \frac{\log \left(P_{\text {error }}^{\text {block }}(\mathcal{P}, W)\right)}{\log (W)} .
$$

This definition describes the asymptotic behavior of error probability with bandwidth, for fixed received power. Since, SNR $\propto 1 / W$, an equivalent definition of low SNR diversity is ${ }^{2}$ :

$$
d_{L} \triangleq \lim _{\text {SNR } \rightarrow 0} \frac{\log \left(P_{\text {error }}^{\text {block }}(\mathcal{P}, \text { SNR })\right)}{\log (\mathrm{SNR})}
$$

From $(2,3)$, we have

$$
d_{L}=r \cdot t \cdot[\kappa-\min \{1, \nu\}]+1-\min \{1, \nu\} .
$$

Hence, we conclude that the decay in error probability is exponential with the product of the number of transmit and receive antennas, $r \cdot t$. Similar to the high SNR regime, the product of the number of transmit and receive antennas comes about as a diversity factor in the low SNR regime. Hence, we conjecture that $r \cdot t$ is a diversity factor for a MIMO channel at any SNR.

From the capacity results in [14], we have seen that receive antennas have greater significance than transmit antennas since, the former effects the linear as well as the sublinear capacity term whereas, the latter effects only the sublinear term. However, since the error probability decays exponentially with $r \cdot t$, the transmit antennas have the same importance as receive antennas in terms of reliability. This emphasizes the importance of multiple transmit antennas in the wideband regime.

Let us now consider channel outage in the low SNR regime. For a block fading channel, outage occurs in a coherence block when the channel matrix is so ill-conditioned that the block mutual information cannot support the target block data rate.

\footnotetext{
${ }^{2}$ We omit the argument of $d_{L}($.$) for simplicity.$
} 
We denote the outage probability as $P_{\text {outage }}$ and present a heuristic computation to show that

$$
P_{\text {error }}^{\text {block }} \sim \mathrm{SNR}^{1-\min \{1, \nu\}} \cdot P_{\text {outage }} \text {. }
$$

Thus, we see that in the low SNR/wideband regime, for rates away from capacity, the error probability is dominated by the outage probability. Hence, like at high SNR, channel outage is the major source for errors even at low SNR.

Heuristic Proof (full proof in [15]): The outage probability can be upper bounded using a training based scheme (This scheme is described in detail in [15]). We directly state the channel model for data transmission (the first $t$ symbols are used for training):

$$
\overrightarrow{\mathbf{y}}_{i}=\mathbf{H}^{\prime} \overrightarrow{\mathbf{x}}_{i}+\overrightarrow{\mathbf{v}}_{i}^{\prime}, \quad i=t+1, \ldots, l,
$$

where, $\mathbf{H}^{\prime}$ has i.i.d $\mathcal{C} N(0,1)$ entries and is perfectly known at the receiver (this is the MMSE channel estimate), $\overrightarrow{\mathbf{v}}_{i}^{\prime}$ is a zero-mean noise vector having the covariance matrix

$$
E\left[\overrightarrow{\mathbf{v}}_{i}^{\prime} \overrightarrow{\mathbf{v}}_{i}^{\prime \dagger}\right]=I_{r}
$$

and, $\left\{\overrightarrow{\mathbf{x}}_{i}\right\}$ are i.i.d complex Gaussian vectors:

$$
\overrightarrow{\mathbf{x}}_{i} \sim \mathcal{C N}\left(0, \frac{f^{*}(\mathrm{SNR})}{t} \cdot I_{t}\right)
$$

where,

$$
f^{*}(\mathrm{SNR})=\mathrm{SNR}^{\min \{1, \nu\}}+o\left(\mathrm{SNR}^{\min \{1, \nu\}}\right) .
$$

Now,

$$
\begin{aligned}
& P_{\text {outage }} \\
& \quad=\operatorname{Pr}\left(I\left(\overrightarrow{\mathbf{x}}_{t+1}, \ldots, \overrightarrow{\mathbf{x}}_{l} ; \overrightarrow{\mathbf{y}}_{t+1}, \ldots, \overrightarrow{\mathbf{y}}_{l} \mid \mathbf{H}^{\prime}\right)<R\right) \\
& \quad \leq \operatorname{Pr}\left(\log \operatorname{det}\left(I_{t}+\frac{f^{*}(\mathrm{SNR})}{t} \mathbf{H}^{\prime}{ }^{\dagger} \mathbf{H}^{\prime}\right)<\frac{R}{l-t}\right) \\
& \quad \leq \operatorname{Pr}\left(\log \left(1+\frac{f^{*}(\mathrm{SNR})}{t} \operatorname{trace}\left(\mathbf{H}^{\prime}{ }^{\dagger} \mathbf{H}^{\prime}\right)\right)<\frac{R}{l-t}\right)(6) \\
& \quad \sim \operatorname{Pr}\left(\chi_{r t}^{2}<\frac{R}{l f^{*}(\mathrm{SNR})}\right) .
\end{aligned}
$$

Equation (5) follows since the mutual information is minimized if $\left\{\overrightarrow{\mathbf{v}}_{i}^{\prime}\right\}$ are i.i.d complex Gaussian [7], [10]. In (6), we use the inequality:

$\operatorname{det}\left(I_{t}+\frac{f^{*}(\mathrm{SNR})}{t} \mathbf{H}^{\prime} \dagger \mathbf{H}^{\prime}\right) \geq 1+\frac{f^{*}(\mathrm{SNR})}{t} \operatorname{trace}\left(\mathbf{H}^{\prime}{ }^{\dagger} \mathbf{H}^{\prime}\right)$.

In (7), $\chi_{r t}^{2}$ represents trace $\left(\mathbf{H}^{\prime} \dagger \mathbf{H}^{\prime}\right)$ and is a chi-squared random variable with $r t$ degrees of freedom. Hence, if we choose the rate in Region B as in (3), we have for low SNR,

$$
\frac{R}{l f^{*}(\mathrm{SNR})} \ll 1 \text {. }
$$

Hence,

$$
\begin{aligned}
& P_{\text {outage }} \sim\left[\frac{R}{l \cdot \mathrm{SNR}^{\min \{1, \nu\}}}\right]^{r t} \\
& \Rightarrow P_{\text {error }}^{\text {block }} \sim \operatorname{SNR}^{1-\min \{1, \nu\}} \cdot P_{\text {outage }} \text {. }
\end{aligned}
$$

\section{CONCLUSIONS}

In this paper, we have computed the error probability for the non-coherent wideband MIMO channel. Our analysis is a more detailed characterization than [12], of the effect on reliability of coherence length, bandwidth and number of transmit and receive antennas. We have shown that error probability decays inversely with the coherence length and exponentially with the product of the number of transmit and receive antennas. This highlights the importance of multiple transmit antennas, besides multiple receive antennas, in the low SNR regime. An interesting observation has been that outage probability dominates the error probability even at low SNR.

\section{ACKNOWLEDGEMENTS}

The authors acknowledge the research grants NSF ANI0335256, NSF CCR 0093349 and HP Award 008542-008.

\section{REFERENCES}

[1] R. G. Gallager, "A Simple Derivation of the Coding Theorem and Some Applications", IEEE Transactions on Information Theory, Vol. 11, No. 1, pp. 3-18, January 1965.

[2] G.J. Foschini, "Layered space-time architecture for wireless communication in a fading environment when using multi-element antennas", Bell Labs Technical Journal, Vol. 1, No. 2, pp. 41-59, 1996.

[3] V. Tarokh, "Space-Time Codes for High Data Rate Wireless Communication: Performance Criterion and Code Construction", IEEE Transactions on Information Theory, Vol. 44, pp. 744-765, March 1998.

[4] T. L. Marzetta and B. M. Hochwald, "Capacity of a mobile multipleantenna communication link in flat fading", IEEE Transactions on Information Theory, Vol. 45, pp. 139-157, January 1999.

[5] I.E. Telatar, "Capacity of multi-antenna Gaussian channels", European Transactions on Telecommunications, 10(6):585-95, Nov/Dec 1999.

[6] B. M. Hochwald and T. L. Marzetta, "Unitary Space-Time Modulation for Multiple-Antenna Communications in Rayleigh Flat Fading”, IEEE Transactions on Information Theory, Vol. 46, pp. 543-564, March 2000.

[7] M. Médard, "The effect upon channel capacity in wireless communications of perfect and imperfect knowledge of the channel", IEEE Transactions on Information Theory, Vol. 46, May 2000.

[8] L. Zheng and D. N. C. Tse, "Communication on the Grassmann Manifold: A geometric approach to the noncoherent multiple-antenna channel", IEEE Transactions on Information Theory, Vol. 48, pp. 359-83, February 2002.

[9] L. Zheng and D. N. C. Tse, "The Diversity-Multiplexing Tradeoff for Non-coherent Multiple Antenna Channels", Allerton Conference 2002, 2002.

[10] B. Hassibi and B. M. Hochwald, "How much training is needed in multiple-antenna wireless links?", IEEE Transactions on Information Theory, Vol. 49, pp. 951-963, April 2003.

[11] L. Zheng and D. N. C. Tse, "Diversity and Multiplexing: A Fundamental Tradeoff in Multiple-Antenna Channels", IEEE Transactions on Information Theory, Vol. 49, pp. 1073-1096, May 2003.

[12] X. Wu and R. Srikant, "MIMO Channels in the Low SNR Regime: Communication Rate, Error Exponent and Signal Peakiness", Information Theory Workshop 2004, San Antonio, Texas, 2004.

[13] L. Zheng, D. N. C. Tse and M. Médard, "Channel Coherence in the low SNR Regime", submitted to IEEE Transactions on Information Theory. Available at http://web.mit.edu/lizhong/www/.

[14] S. Ray, M. Médard and L. Zheng, "Wideband Non-coherent MIMO Capacity, ISIT 2005, Adelaide, Australia, Sep. 4-9, 2005.

[15] S. Ray, M. Médard and L. Zheng, "On Non-coherent MIMO Channels in the Wideband Regime: Capacity and Reliability", submitted to IEEE Transactions on Information Theory. ArXiv cs.IT/0603018. 